\title{
Traffic Vertical Signposting: Materials Characterization and Structural Numerical Simulation
}

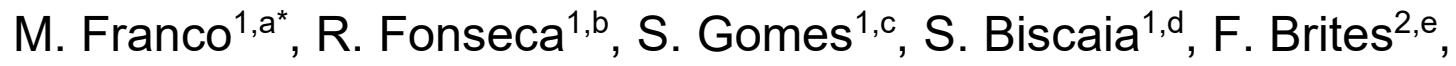 \\ P. Pascoal-Faria ${ }^{1,3, f}$ and A. Mateus ${ }^{1, g}$ \\ ${ }^{1}$ Centre for Rapid and Sustainable Product Development, Polytechnic Institute of Leiria, Rua de \\ Portugal, Marinha Grande, 2430-028, Portugal \\ ${ }^{2}$ Department of Mechanical Engineering, Polytechnic Institute of Coimbra, Rua Pedro Nunes, \\ 3030-199 Coimbra,Portugal \\ ${ }^{3}$ Department Mathematics, School of Technology and Management, Polytechnic Institute of Leiria, \\ Rua General Norton de Matos, Apartado 4133, 2411-901 Leiria - Portugal, Portugal \\ amargarida.franco@ipleiria.pt, bana.r.fonseca@ipleiria.pt, csara.gomes@ipleiria.pt, \\ dsara.biscaia@ipleiria.pt, efernando.mp.brites@gmail.com, fpaula.faria@ipleiria.pt, \\ gartur.mateus@ipleiria.pt
}

Keywords: polymer composites, injection moulding, vertical traffic signs, numerical analysis, structural design.

\begin{abstract}
The existing metallic solutions used for vertical traffic signs are associated with higher costs and environmental issues due to their manufacturing and degradation, when compared with polymeric solutions. Thus, the development of vertical signs considering the injection from polymeric materials in order to overcome problems related with sustainability, maintenance costs, and to achieve higher resistance to corrosion assumes nowadays an important role. The use of ecofriendly and innovative products considering the industrial waste combined with synthetic polymers performing the appropriate mechanical properties, can also be studied to find out new solutions that allow to solve the aforementioned problems. Additionally, these innovative vertical signs can contribute to avoid vandalism events related with theft and graffiti activities. This work presents the prior materials investigation and the structural design of vertical signs that are intended to be produced through polymer injection. Three main steps were considered: i) materials research, ii) materials characterisation through the analysis of polycarbonate resin isolated and in different sets of mixtures with different concentrations through tensile testing and static water contact angle measurements to find the optimal material composition; and iii) structural numerical simulation considering polycarbonate resin and using the current standard EN 12899-1 [1] to compute wind resistance, temporary and permanent deflections. Both experimental and numerical results led to an optimized proposal of the vertical signposting structural design.
\end{abstract}

\section{Introduction}

At present, problems related with sustainability, maintenance, costs and corrosion are at the top of government priorities. In fact, corrosion is a very sensitive issue in countries with a very large coast border like Portugal, making the search for new solutions to replace the traditional traffic sings by non-corrosive materials, a topic of major interest.

The high tenacity of polymers in terms of corrosion resistance, providing a longer life and a higher elastic recovery when compared with metals, is well known. Characteristics as lightness, easy handling and transport, are major improvements compared with the existing metallic signs. Its current metallic nature makes them expensive and environmentally compromising due to the high energy costs associated with their manufacturing and degradation, not to mention the serious problems of corrosion and oxidation associated, which contribute to their shortened lifespan. In addition, questions related to traffic signs theft and vandalism motivated by their metallic nature are a serious concern, since this can lead to an increase in unsafe driving behaviors. Additionally, self- 
cleaning properties, derived from the slightly hydrophilic nature of the polymer composites used, can prevent graffiti activities and oils pollution deposition. The possibility of some polymers to acquire photoluminescence properties can enable the traffic sign to be seen by wild animals during the night period empowering it to protect them.

This study presents the development of a traffic sign plate made of polymer to fill the existing need in temporary and permanent vertical traffic signs. The final solution aims an eco-friendly and innovative device which can incorporate materials coming from industrial waste, together with synthetic polymers performing the appropriate mechanical properties, wettability behavior, wind resistance, temporary/permanent deflections, and that can be produced in a large scale through injection. Along with the mentioned improvements, the traffic sign proposed in this work will display photoluminescence characteristics providing the existence of light emission after the absorption of light energy. Several sets of composites were produced and characterized by tensile testing and static water contact angle measurements to find the optimal material composition for the advanced and smart traffic signs manufacturing suggested here. The selected samples were submitted to static water contact angle assays, tensile tests and structural numerical simulation studies.

\section{Materials and Methods}

\section{Materials}

The adopted materials/mixtures selected for the production of the specimens, namely the concentrations of each material in the mixture, reference and supplier are presented in Table 1 and Fig.1. Polycarbonate (PP), Polycarbonate with Acrylonitrile styrene acrylate (PC/ASA) and Polycarbonate with glass fiber (PP/GF) were obtained from RESINEX, TAROPLAST and LG CHEM, respectively. Three photoluminescent pigments were employed: Lumilux ${ }^{\circledR}$ SN-F2, Lumilux ${ }^{\circledR}$ N-FG, from HORQUIM and LumiNova ${ }^{\circledR}$ G-300 which came from ZEUS QUÍMICA.

Table 1 - Tested samples.

\begin{tabular}{|c|c|c|c|}
\hline Sample & Material & Reference & Supplier \\
\hline $\mathrm{PC}$ & Polycarbonate & CALIBRETM 303EP-31 & RESINEX \\
\hline PCV40_PCR60 & PC virgin/ PC recycled mixture (40:60) & CALIBRETM 303EP-31 & RESINEX \\
\hline PCV60_PCR40 & PC virgin/ PC recycled mixture $(60: 40)$ & CALIBRETM 303EP-31 & RESINEX \\
\hline PP70_GF30 & $\begin{array}{c}\text { Commercial mixture of polypropylene } \\
\text { with (30\%) glass fibre }\end{array}$ & HAIPLEN H50 G6 BA & TAROPLAST \\
\hline PC_ASA & $\begin{array}{l}\text { Commercial mixture of polycarbonate } \\
\text { with Acrylonitrile styrene acrylate }\end{array}$ & LUPOY EU5000G & LG CHEM \\
\hline PC80_PL20 & $\begin{array}{c}\text { Polycarbonate / photoluminescent } \\
\text { pigment (LumiNova }{ }^{\circledR} \text { G-300) mixture } \\
(80: 20)\end{array}$ & $\begin{array}{l}\text { PC: CALIBRETM 303EP-31 } \\
\text { PL: LumiNova }{ }^{\circledR} \text { G-300 }\end{array}$ & $\begin{array}{l}\text { RESINEX, } \\
\text { ZEUS QÍMICA }\end{array}$ \\
\hline PC80_PLB20 & $\begin{array}{c}\text { Polycarbonate / photoluminescent } \\
\text { pigment (Lumilux }{ }^{\circledR} \text { SN-F2) mixture } \\
(80: 20)\end{array}$ & $\begin{array}{l}\text { PC: CALIBRETM 303EP-31 } \\
\text { PL: Lumilux® SN-F2 }\end{array}$ & $\begin{array}{l}\text { RESINEX, } \\
\text { HORQUIM }\end{array}$ \\
\hline PC80_PLA20 & $\begin{array}{l}\text { Polycarbonate /photoluminescent } \\
\text { pigment (Lumilux }{ }^{\circledR} \text { N-FG) mixture } \\
(80: 20)\end{array}$ & $\begin{array}{l}\text { PC: CALIBRETM 303EP-31 } \\
\text { PL: Lumilux } ® \text { N-FG }\end{array}$ & $\begin{array}{l}\text { RESINEX, } \\
\text { HORQUIM }\end{array}$ \\
\hline
\end{tabular}

PC - Polycarbonate, PCV - Polycarbonate virgin, PCR - Polycarbonate recycled, PL - Photoluminescent, PP Polypropylene, GF - Glass fibers, ASA - Acrylonitrile Styrene Acrylate. 


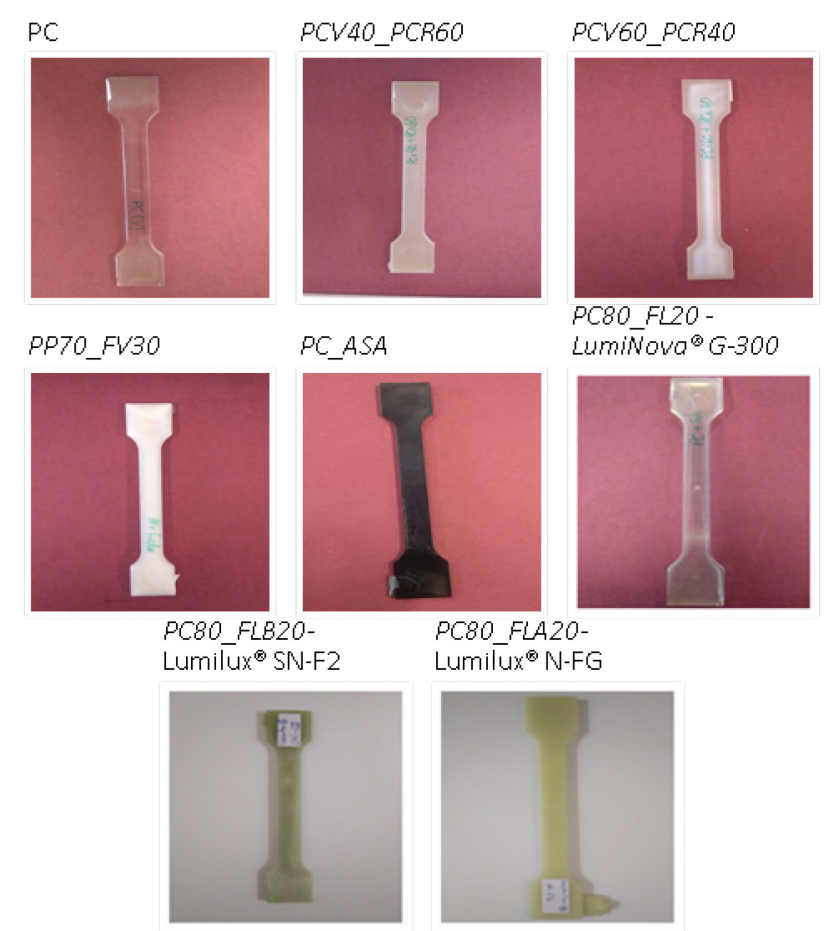

Figure 1 - Specimens used for characterization tests.

\section{Methods}

\section{Contact Angle Measurement}

The contact angle assay is a quantitative measure of the wettability of a solid by a liquid, in which higher surface energies being associated with lower contact angles. The wettability of the specimens was evaluated by static contact angle measurement on a Theta Lite optical tensiometer (Attension - Biolin Scientific, Finland). A sessile drop methodology was followed. A water droplet was poured on the surface of the samples and the contact angle was measured by OneAttension 1.0 software (Attension). Water was the liquid phase. All assays were performed in triplicate.

\section{Mechanical properties}

The mechanical properties of the developed samples were determined performing tensile tests, using an Instron 4500 Universal Machine, according to the standard ASTM D3039. Tensile test specimens were prepared according to the standard ISO 527-2. Tensile modulus was estimated from the initial slope of the stress-strain curve using the linear regression method. The average and standard deviations were determined using at least 5 specimens per condition.

\section{Structural Numerical Simulation}

\section{Numerical model}

The structural simulation was carried on considering the circular sign plate illustrated in Fig. 2. The design was performed to determine the minimum thickness of the sign plate. The threedimensional model was implemented using tetrahedron finite elements with 20 nodes. A total of 38046 elements were used (see Fig. 3). The bolts were designed with beam elements, fixed at extremities and bonded to sign plate using the multiple point contact (MPC). The sign plate was modelled considering the materials presented in the previously (see Fig. 2) and bolts were considered to be made of steel with a tensile strength of $250 \mathrm{MPa}$ and a Young's modulus of $200 \mathrm{GPa}$. 


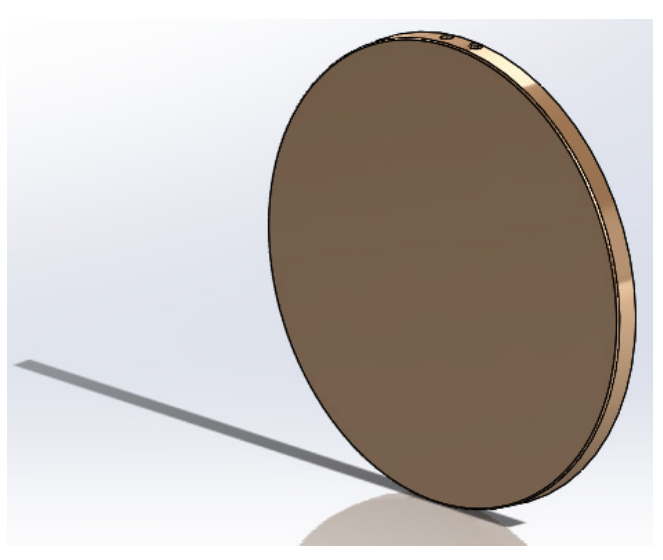

(a)
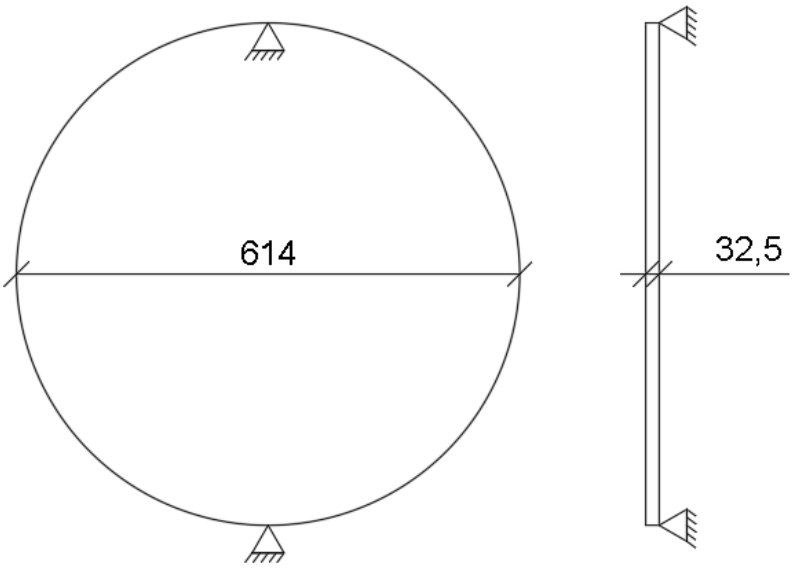

(b)

Figure 2 - Traffic sign: (a) overview; and structural scheme (mm).

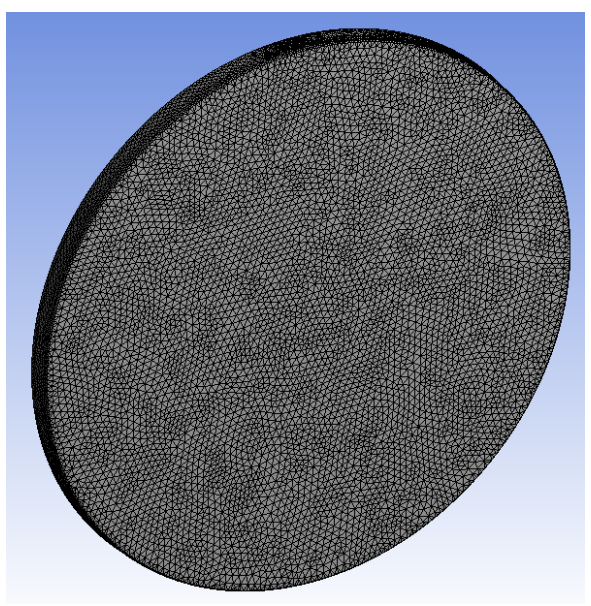

(a)

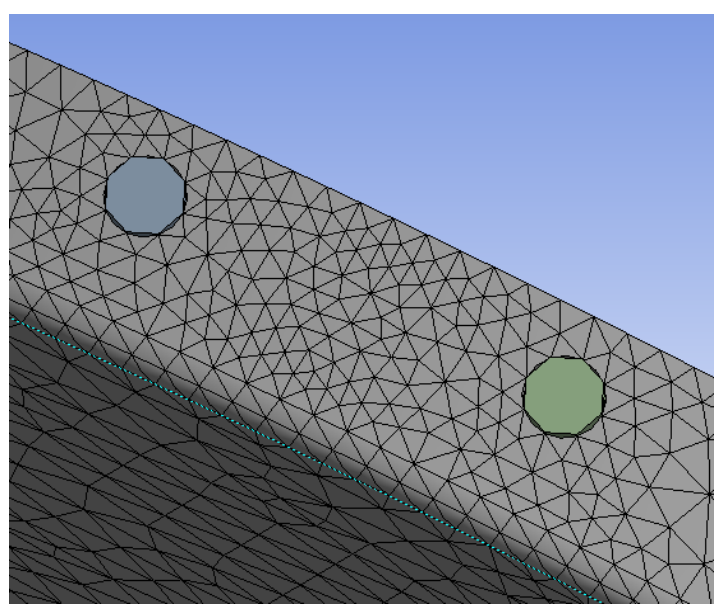

(b)

Figure 3 - Finite element mesh: (a) sign overview; and (b) bolts detail.

\section{Structural analysis}

The structural analysis was performed according to the standard EN 12899-1 that regards the following verifications: Ultimate Limit State (ULS), Service Limit States (SLS) - temporary deflections and permanent deflections. The partial safety factors adopted for the ULS and the permanent deflections are presented in Table 2, whereas a reduction factor of 0.56 was considered for temporary deflections. The characteristics values used for the ULS and SLS permanent deflections are presented in Table 3 and illustrated in Figure 4. The wind load was multiplied by a shape factor of 1.20 .

Table 2 - Partial safety factors for loads $(\gamma \mathrm{F})$ and materials $(\gamma \mathrm{m})[1]$.

\begin{tabular}{cc}
\hline Load/Material & Partial safety factor \\
\hline Wind and point loads & 1.35 \\
\hline Dead load & 1.20 \\
\hline Plastic & 1.80 \\
\hline
\end{tabular}

Table 3 - Characteristic values of loads [1].

\begin{tabular}{cc}
\hline Load & Partial safety factor \\
\hline Wind WL6 $\left(\mathrm{kN} . \mathrm{m}^{-2}\right)$ & 1.20 \\
\hline Point load PL1 $(\mathrm{kN})$ & 0.15 \\
\hline
\end{tabular}




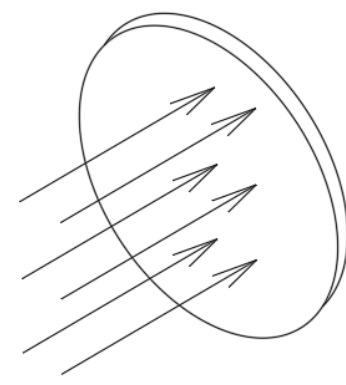

(a)

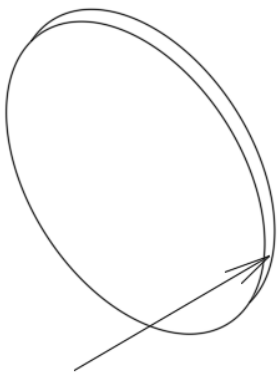

(b)

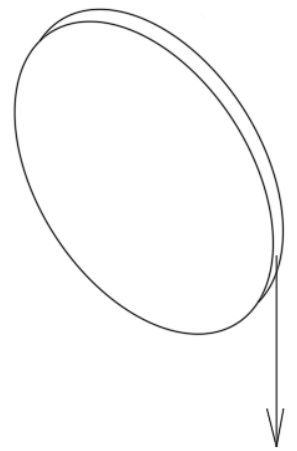

(c)

Figure 4 - Types of loads application: (a) pressure, (b) horizontal point load; and (c) vertical point load [1].

\section{Results and Discussion}

\section{Surface Wettability of the selected materials}

The measured contact angles are shown in Fig. 5. The measurement of a substance or material contact angle $(\theta)$ reflects the wettability of the material by the test liquid and refers to the angle formed by the liquid when it comes in contact with the solid test material surface. The value of the calculated contact angle depends mainly on the affinity forces between the liquid and the solid. When forces are high, the contact angle is less than 90 degrees and it results in the ability of the liquid to wet the solid material. From Fig. 5 it is possible to see that the mixtures are hydrophilic. The contact angle established between a drop of water and the different studied materials is less than $90^{\circ}$. Moreover, the weak PC's hydrophilia was slightly increased by the incorporation of any of the photoluminescent pigments employed $(85.65+/-0.72$ against $76.84+/-0.71,62.81+/-3.06$ and 58.64+/-5.61, belonging to PC80_PL20, PC80_PLB20 and PC80_PLA20, respectively). Thus, a low affinity with grease, which is the main component of the polluting gases and vapors of the road, is ensured. This characteristic results from the high hydrophobia of these pollutants. The hydrophilic characteristic of the chosen materials will also contribute to prevent vandalism activities. Actually, the hydrophobic nature of some of the pigments used in graffiti paint is not favorable for the required adhesion on the studied polymers. In addition, these materials are able to repel dust and contaminants through the creation of a uniform water film onto the surface.

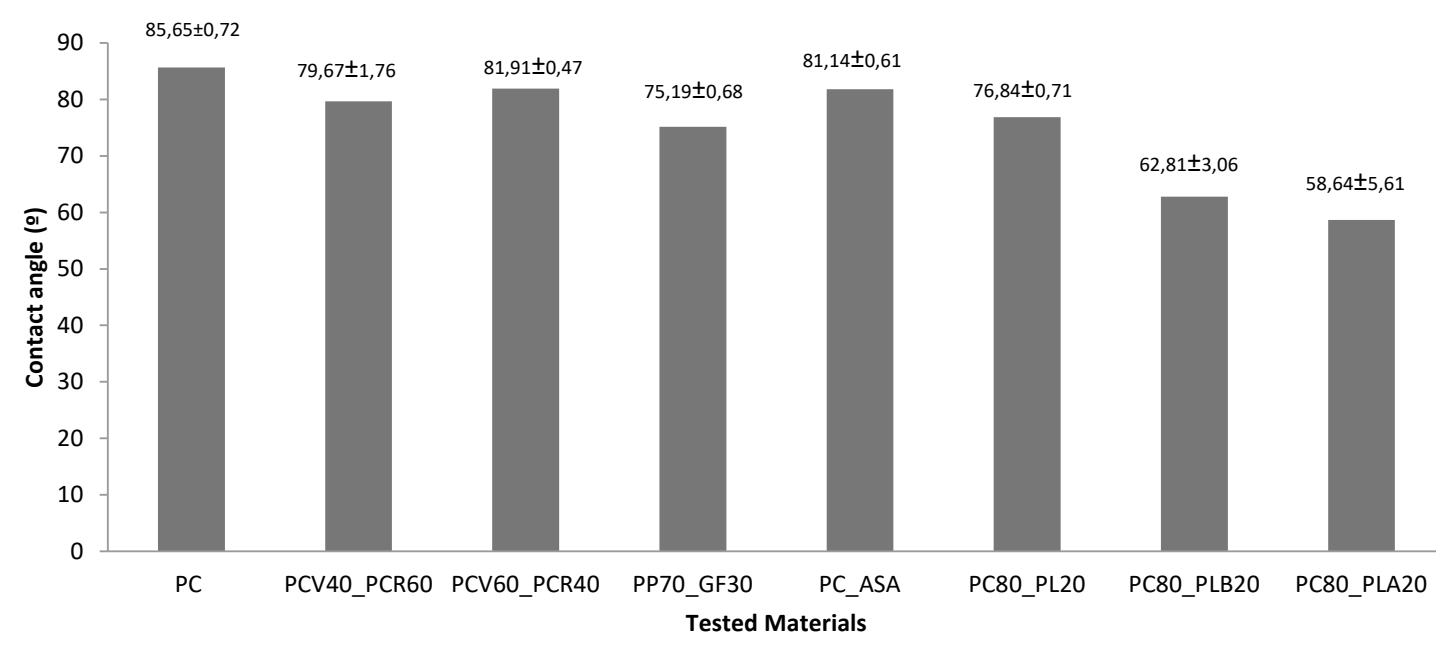

Figure 5 - Static water contact angle of the specimens. 


\section{Tensile tests}

To characterize the mechanical performance of the developed samples, tensile tests were performed. Table 4 summarizes the obtained results regarding the maximum tensile strength and tensile modulus. All results are expressed as means \pm standard deviation.

Comparing pure PC and PCV_PCR mixtures is possible to observe similar values of maximum tensile strength. On the other hand, with the increase of recycled content the Young modulus decreases, PC undergoes a slight degradation during reprocessing. This decrease is in agreement with the observed by other authors $[2,3]$ and could be correlated to the decreases in impact strength and molecular weight.

Concerning the mechanical tests of PC with different photoluminescent pigments is possible to observe a small decrease of the tensile strength in all the samples and an increase of the Young modulus values with the addition of lumilux photoluminescent pigments. This values can be related with the interfacial adhesion and bonding between the matrix and photoluminescent powder. Mechanical properties of PP_FV and PC_ASA samples are in accordance to mechanical ASTM \& ISO characteristics of materials technical data.

Table 4 - Average results of mechanical tensile tests.

\begin{tabular}{ccc}
\hline Material & Tensile Strength (MPa) & Modulus of Elasticity (MPa) \\
\hline PC & $58,35 \pm 0,54$ & $2334,88 \pm 60,27$ \\
\hline PCV40_PCR60 & $58,88 \pm 0,53$ & $2140,53 \pm 71,46$ \\
\hline PCV60_PCR40 & $57,42 \pm 0,48$ & $2062,08 \pm 45,88$ \\
\hline PP70_FV30 & $90,64 \pm 1,55$ & $6773,05 \pm 103,31$ \\
\hline PC_ASA & $48,97 \pm 0,77$ & $2126,08 \pm 59,62$ \\
\hline PC80_PL20 & $57,34 \pm 0,45$ & $2033,27 \pm 50,32$ \\
\hline PC80_PLB20 & $52,15 \pm 2,33$ & $2435,55 \pm 67,35$ \\
\hline PC80_PLA20 & $55,30 \pm 1,66$ & $2415,40 \pm 91,85$ \\
\hline
\end{tabular}

\section{Structural numerical simulation}

The results presented herein were computed for a thickness of $4.5 \mathrm{~mm}$ and PC+ASA. For the ULS (see Fig. 6) tensile stresses reach values close to $40.90 \mathrm{MPa}$ near the supports area. As expected, there is a stress concentration close to supports due to the geometry, whereas in the remaining area stresses are below the maximum accepted elastic limit stress (27.8 $\mathrm{MPa})$. The standard accepts the existence of plastic areas when ductile materials are considered.

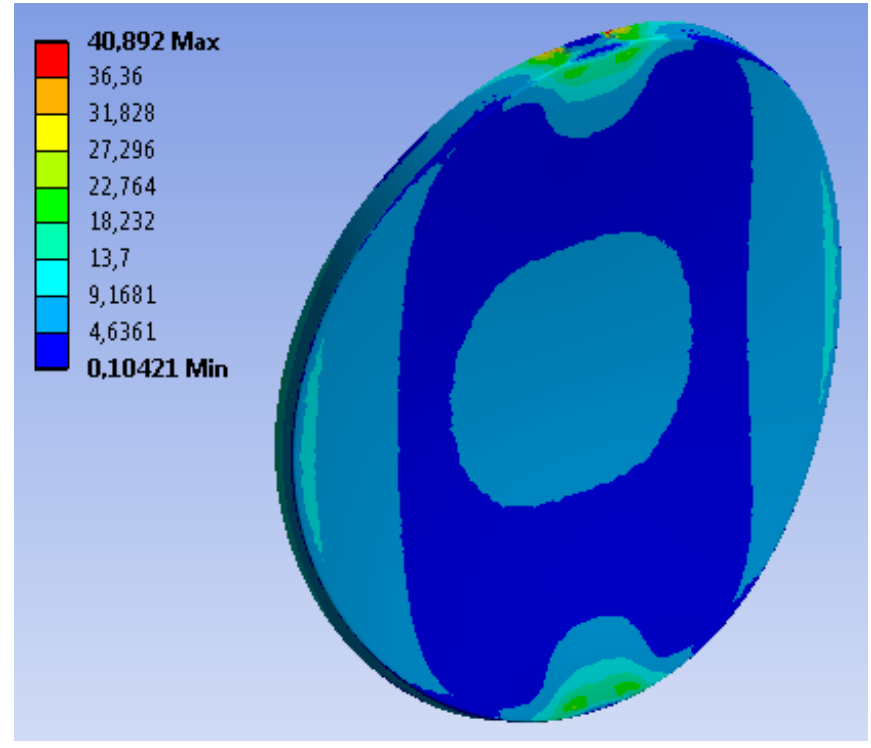

(a)

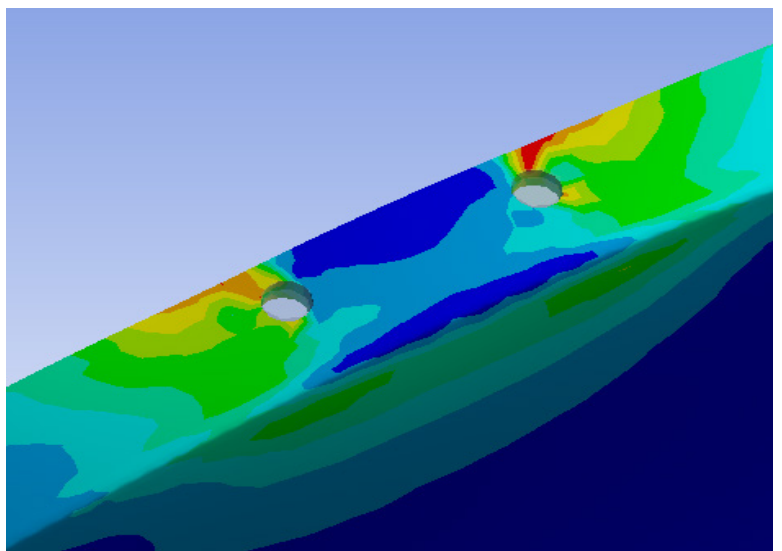

(b)

Figure 6 - Von Mises tensile strength (MPa) for the ULS: (a) signal plate overview; and (b) details close to bolts. 
The numerical analysis regarding the SLS for temporary deflections verification, allows finding that the maximum deflection occurs at extremities and reaches values close to $15.35 \mathrm{~mm}$ (see Fig. 7). This value respects the maximum temporary deflection class TDB5 that accepts having a bending deflection of $50 \mathrm{~mm} \cdot \mathrm{m}^{-1}$.

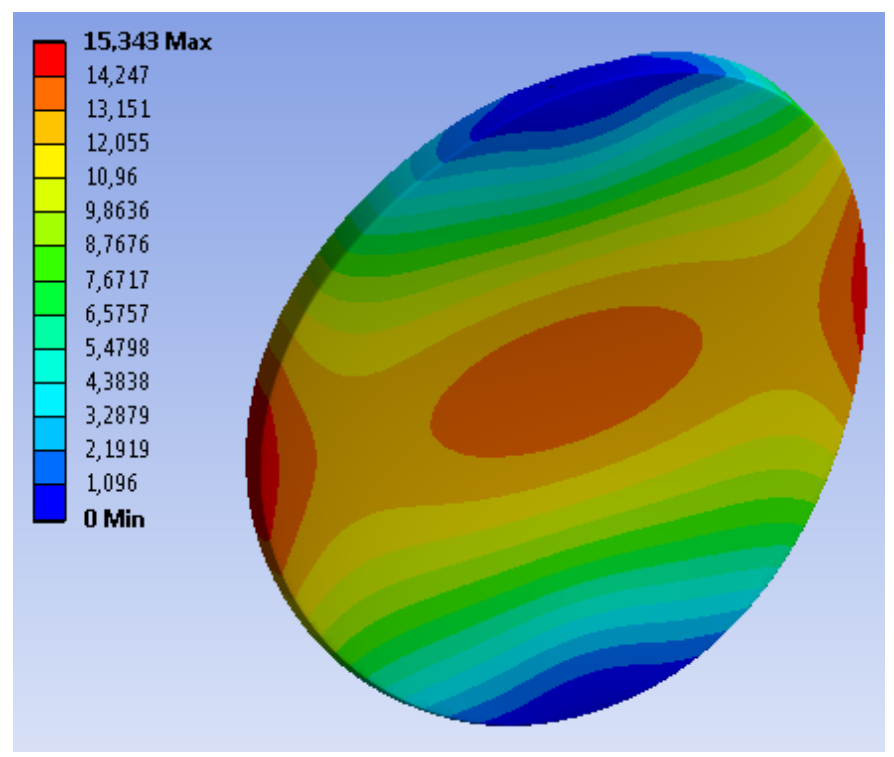

(a)

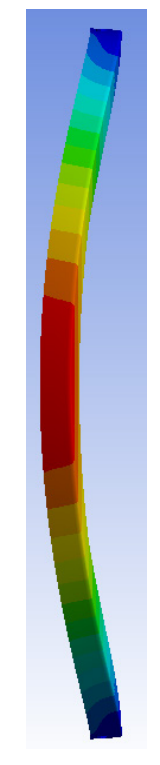

(b)

Figure 7 - Maximum displacement (mm) for the SLS - temporary deflections: (a) signal plate overview; and (b) longitudinal bending.

The permanent deflection regarding the SLS was assessed for wind (see Fig. 8), horizontal point load (see Fig. 9) and vertical point load (see Fig. 10). Results showed that for all the three simulations the von Mises stresses are below to the elastic limit stress $(27.8 \mathrm{MPa})$, except around the bolts, which is an area of stress concentration. Herein, stresses should not exceed the elastic limit. Therefore, a strengthening of the bolts area can be performed to reduce the stress concentration at the bolts area.

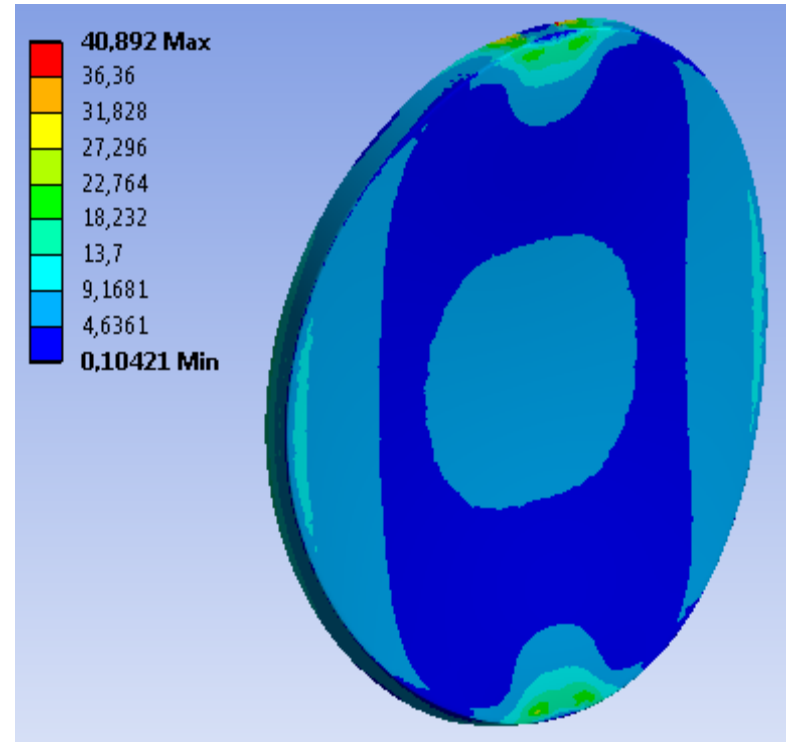

(a)

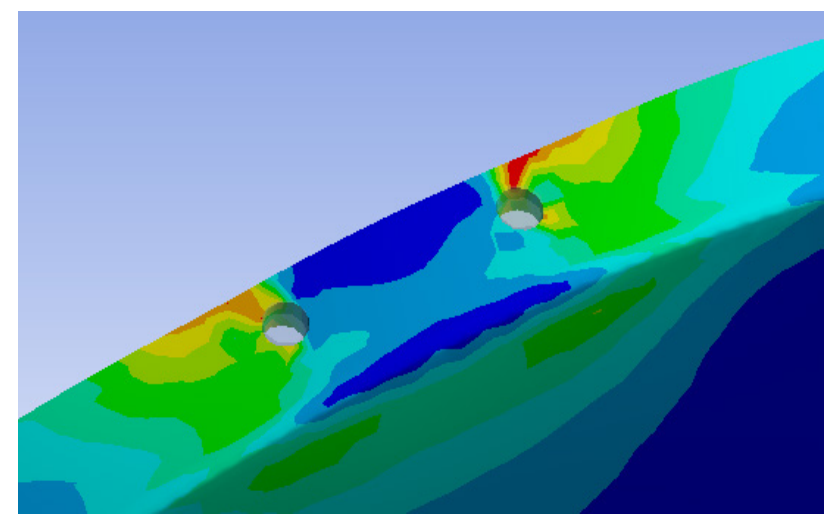

(b)

Figure 8 - Von Mises tensile strength (MPa) for the SLS - permanent deflections - considering wind pressure: (a) signal plate overview; and (b) details close to bolts. 


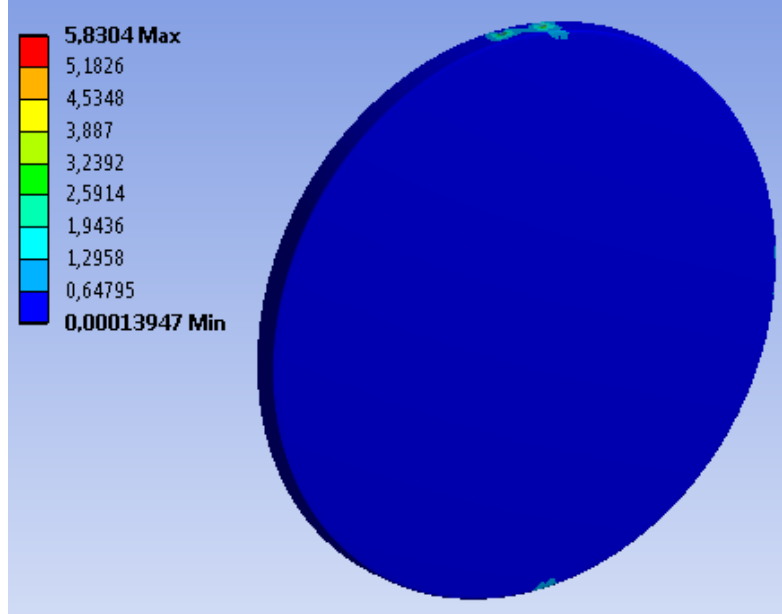

(a)

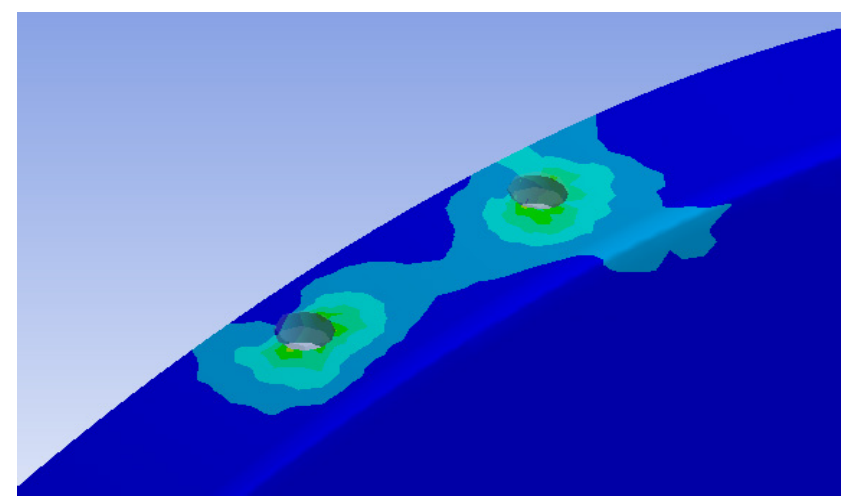

(b)

Figure 9 - Von Mises tensile strength (MPa) for the SLS - permanent deflections - considering horizontal point load: (a) signal plate overview; and (b) details close to bolts.

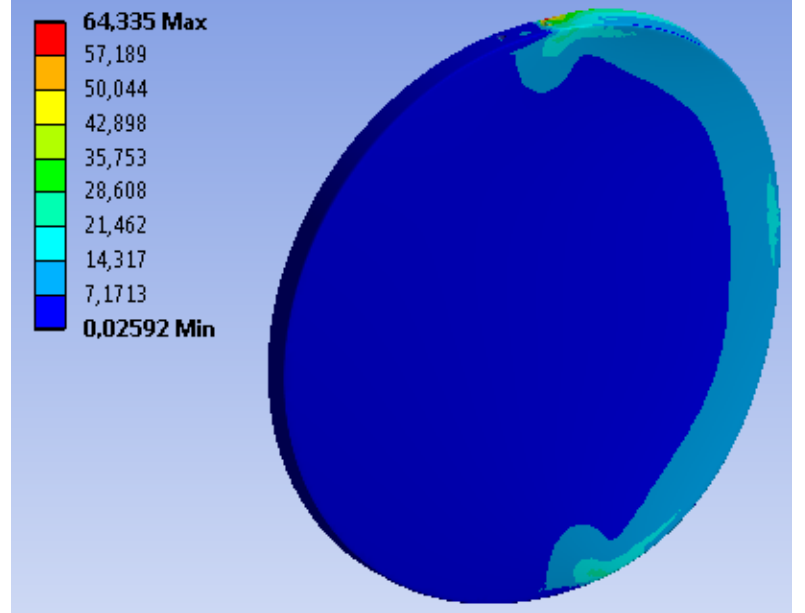

(a)

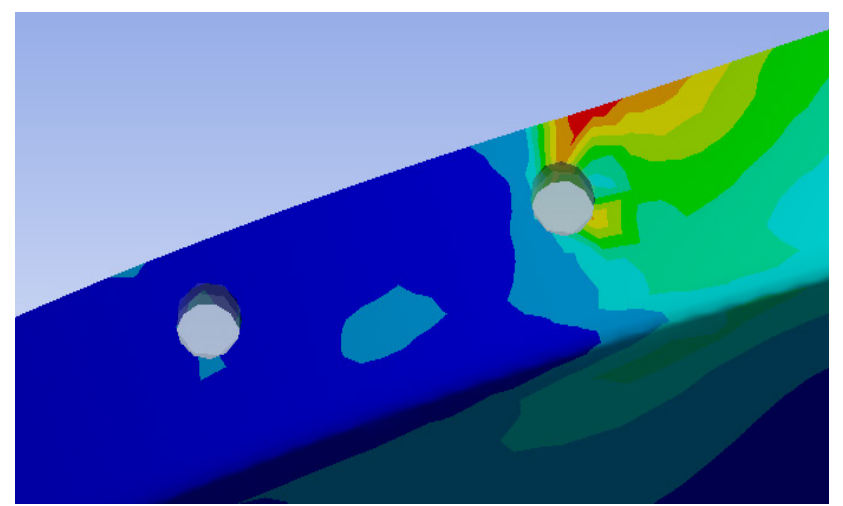

(b)

Figure 10 - Von Mises tensile strength (MPa) for the SLS - permanent deflections - considering vertical point load: (a) signal plate overview; and (b) details close to bolts.

\section{Conclusions}

This paper presented the development of a vertical traffic sign using polymer materials. Results showed that it is possible to manufacture vertical traffic signs using polymer materials through injection that entirely meet the requirements of the above referred European standard.

The contact angle measurements have shown that all materials present hydrophilic properties, meaning that these materials can repel dust and prevent vandalism activities. The tensile tests allowed to verify that tensile strength decreases and the Young's modulus increase when recycle content and photoluminescent pigments are used.

The numerical model was performed for the PC+ASA. Results showed that the critical verification is related with the SLS for temporary deflections and that all requirements according to the standard EN 12899-1 are ensured for sign plate thickness of $4.5 \mathrm{~mm}$.

\section{Aknowlegements}

This work was supported by the project POCI-01-0247-FEDER-010797from the Agency for Competitiveness and Innovation (IAPMEI). In addition, the authors acknowledge the funding from the project UID/Multi/04044/2013 of the Portuguese Foundation for Science and Technology (FCT) and PAMI - ROTEIRO/0328/2013 ( $\mathrm{N}^{\circ}$ 022158) 


\section{References}

[1] EN 12899-1: Fixed, vertical road traffic signs - Part 1: Fixed signs, European Committee for standardization, Brussels, November 2007.

[2] J.M. Péreza, J.L. Vilas a, J.M. Lazaa, S. Arnáizb, F. Mijangosa, E. Bilbaoc, M. Rodrígueza, L.M. Leóna, 2010. Effect of reprocessing and accelerated ageing on thermal and mechanical polycarbonate properties. Journal of Materials Processing Technology 210 (2010) 727-733.

[3] J.I. Eguiazabal, J. Nazabal. Effect of reprocessing on the properties of bisphenol-A polycarbonate. Eur. Polym. J. 25, (1989) 891-893.

[4] Fabrication of high aspect ratio nanopillars and micro/nano combined structures with hydrophobic surface characteristics by injection molding / Applied Surface Science 427 (2018) 854-860

[5] Engineering Polymers thermoplastics and polyurethanes, A Design Guide, Copyright (C) 1995, Bayer Corporation 